ORIGINAL ARTICLE

\title{
Reduced protein synthesis in schizophrenia patient-derived olfactory cells
}

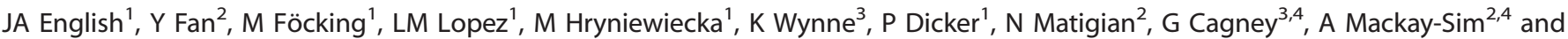 \\ DR Cotter ${ }^{1,4}$
}

Human olfactory neurosphere-derived (ONS) cells have the potential to provide novel insights into the cellular pathology of schizophrenia. We used discovery-based proteomics and targeted functional analyses to reveal reductions in 17 ribosomal proteins, with an $18 \%$ decrease in the total ribosomal signal intensity in schizophrenia-patient-derived ONS cells. We quantified the rates of global protein synthesis in vitro and found a significant reduction in the rate of protein synthesis in schizophrenia patient-derived ONS cells compared with control-derived cells. Protein synthesis rates in fibroblast cell lines from the same patients did not differ, suggesting cell type-specific effects. Pathway analysis of dysregulated proteomic and transcriptomic data sets from these ONS cells converged to highlight perturbation of the elF2a, elF4 and mammalian target of rapamycin (mTOR) translational control pathways, and these pathways were also implicated in an independent induced pluripotent stem cell-derived neural stem model, and cohort, of schizophrenia patients. Analysis in schizophrenia genome-wide association data from the Psychiatric Genetics Consortium specifically implicated elF2a regulatory kinase EIF2AK2, and confirmed the importance of the elF2a, elF4 and mTOR translational control pathways at the level of the genome. Thus, we integrated data from proteomic, transcriptomic, and functional assays from schizophrenia patient-derived ONS cells with genomics data to implicate dysregulated protein synthesis for the first time in schizophrenia.

Translational Psychiatry (2015) 5, e663; doi:10.1038/tp.2015.119; published online 20 October 2015

\section{INTRODUCTION}

Schizophrenia is among the most disabling of human diseases, with poorly understood pathophysiology. ${ }^{1}$ Many cellular and molecular phenomena have been described in neurons of schizophrenic patients, mostly based on post-mortem, neuroimaging and pharmacological data; however, there is still no clear understanding of the cellular and molecular mechanisms underlying the disease. One of the major challenges has been accessing appropriate cells, tissues and animal models that are relevant to the disease pathology. We reasoned that protein expression changes in olfactory neurosphere-derived (ONS) cells may provide novel insights into cellular processes that are dysregulated in schizophrenia.

Patient-derived neural cell models of schizophrenia such as those derived from nasal biopsy of the olfactory mucosa, used here, do not require genetic reprogramming and can be obtained from adults with complex genetic disorders. ${ }^{2,3}$ Research in schizophrenia patient-derived olfactory cells has already revealed insights into specific microRNA effects that are in keeping with the molecular changes associated with schizophrenia, ${ }^{4-6}$ as well as disease-associated alterations of cell cycle, cellular adhesion and migration. ${ }^{7,8}$ Disease-associated alterations in migration, along with dysregulated cytoskeletal genes and proteins, were also observed in neural progenitor cells generated from schizophreniaderived induced pluripotent stem cells (iPSCs). ${ }^{9}$ Our objective was to identify disease-associated cellular processes in schizophrenia patient-derived ONS cells. Our strategy was to use discoverybased protein expression profiling to identify significantly altered processes and pathways and examine those altered pathways at a functional level.

\section{MATERIALS AND METHODS}

For more detailed information, please refer to extended experimental procedures in Supplementary Information.

\section{Human ONS cells}

To identify dysregulated cellular pathways, olfactory mucosa biopsies were obtained from schizophrenia patients $(n=9)$ and healthy age- and sexmatched control $(n=9)$ patients (Supplementary Table 1). Olfactory biopsies were dissociated and cultured as neurospheres in epidermal growth factor and basic fibroblast growth factor, after which the patientand control-derived ONS cell lines were grown under standard conditions, with all experiments performed at passages $5-11$, as previously described. ${ }^{8,10}$

\section{Mass spectrometry}

For proteomic analysis of the ONS cells using label-free liquid chromatography-mass spectrometry (LC-MS) ${ }^{11} 1 \mu \mathrm{g}$ of protein from each ONS cell line $(n=18)$ was run in triplicate on a Thermo Scientific (World wide vendor http://www.thermoscientific.com) LTQ ORBITRAP XL mass spectrometer. Label-free quantification was performed using Max Quant (V1.3.0.2; www.maxquant.org). Raw mass spectrometry (MS) files used in this experiment have been uploaded to the proteomics data repository PRIDE to facilitate data dissemination. For targeted MS of ribosomal proteins, schizophrenia or control samples were pooled and injected in triplicate on a Thermo Q-Exactive. Peptides were targeted using a mass

\footnotetext{
${ }^{1}$ Department of Psychiatry, University College of Dublin School of Biomolecular \& Biomedical Science, Royal College of Surgeons in Ireland, Dublin, Ireland; ${ }^{2}$ Eskitis Institute for Drug Discovery, Griffith University, Brisbane, QLD, Australia and ${ }^{3}$ University College of Dublin School of Biomolecular \& Biomedical Science, Dublin, Ireland. Correspondence: Professor DR Cotter and Dr JA English, Department of Psychiatry, Royal College of Surgeons in Ireland, Smurfit Building, Beaumont Hospital, Beaumont, Dublin 9, Ireland.

E-mail: drcotter@rcsi.ie or janeenglish@rcsi.ie

${ }^{4}$ These authors contributed equally to this work.

Received 2 April 2015; revised 16 June 2015; accepted 8 July 2015
} 
selective MS1 single-ion monitoring scan at a resolution of 35000 (for peptide isolation list see Supplementary Dataset 1). The Skyline software package (v2.1.0.4936; https://skyline.gs.washington.edu) was used for MS1 filtering ${ }^{12}$ and retention time prediction ${ }^{13}$ of target peptides (Supplementary Figure 1a). Western blotting for elF2a, RPL13A and RPL18A was used to confirm the above changes in schizophrenia and control ONS cells (Supplementary Figure 1b). Please refer to online methods for further details.

Protein translation assay in ONS cells and fibroblasts

Global protein synthesis was quantified in schizophrenia- and control-derived ONS cells, and fibroblasts from the same individuals. The methionine analog, L-homopropargylglycine (HPG), was incorporated into newly synthesized proteins in culture and was labeled with a fluorescent tag using coppercatalyzed azide-alkyne cycloaddition, according to the manufacturer's protocol ('Click-IT', Invitrogen, Carlsbad, CA, USA, Supplementary Methods). Experiments were performed on all nine schizophrenia patient-derived and nine control-derived ONS cell lines, at three HPG concentrations $(25,50$ or $100 \mu \mathrm{m})$, at four time points (1, 2, 4 or $8 \mathrm{~h})$. Duplicate plates were used for each time point. Differences in protein synthesis between schizophrenia- and control-derived cells were tested in a three-way analysis of variance (ANOVA) with disease status, HPG concentration and exposure time as main effects. Cell area was quantified with automated imaging and analysis using a fluorescent cytoplasmic stain (CellMask, Invitrogen).

\section{Statistical and bioinformatics analysis}

Statistical analysis of label-free LC-MS/MS data was performed in Perseus (V1.3.0.4; www.maxquant.org) and SIMCA-P+ (Version 13.0; Umetrics, Crewe, UK), as detailed in extended Supplementary Methods. Briefly, to identify significant expression changes at the protein level, a Student's t-test was applied at a $5 \%$ threshold, followed by a permutation-based false discovery rate (FDR) at a 10\% threshold (Figure 1). As the objective of our study was to identify coordinated changes in pathways and cellular processes at the level of the proteome, we performed clustering analysis and pathway analysis on the full list of proteins identified as differentially expressed proteins by Student's $t$-test $(P<0.05)$. Principle component analysis and Volcano plots were generated and performed in Perseus (V1.3.0.4), and Partial Least Squares-Discriminant Analysis (PLS-DA) was performed in SIMCA-P+ (Version 13.0; Umetrics). For investigations of potentially confounding effects of cigarette smoking and antipsychotic drug exposure on protein expression, please refer to online methods. Ingenuity pathway analysis was performed on statistically significant proteins and mRNA transcripts as per supplier's instructions (http://www. ingenuity.com/), detailed in Supplementary Methods.

Testing for associations with schizophrenia in genome-wide association data

Single gene-based analysis was tested on the differentially expressed mRNA transcripts and proteins in the elF2 signaling pathway (Table 1). Genome-wide association results were available from the Psychiatric Genetic Consortium. ${ }^{14}$ A gene-based test of the elF2 signaling pathway was performed using the VEGAS software (http://gump.qimr.edu.au/ VEGAS/), and a second analysis of gene sets of EIF2 signaling ( $n=48$ genes), mammalian target of rapamycin (mTOR) signaling ( $n=40$ genes) and EIF4 signaling ( $n=33$ genes) was performed for enrichment with schizophrenia. The enrichment of the gene sets was tested using a competitive test of enrichment, GSEA v2.0 (ref. 15) as detailed in Supplementary Methods.

\section{RESULTS}

Proteomic profiling reveals reduced expression of ribosomal proteins in schizophrenia

We measured the relative expression levels of 859 proteins identified across the 18 ONS samples, using label-free quantitative MS (Supplementary Data set 2). Whereas global protein expression was consistent across all samples (Figure 1a), we found 102 proteins to be differentially expressed between groups, 56 showing increased and 46 decreased expression in patientderived cells (Figure 1b; Data set 2). Fifteen proteins remained significantly differentially expressed following permutation-based FDR, nine showing increased and six decreased expression. To identify co-regulated proteins and disease-associated pathways, we used the results from the low-stringency analysis (102 significant proteins @ $P<0.05$; Data set 2 ) for our clustering and pathway analysis. Principle component analysis of the differentially expressed proteins revealed a clear separation between schizophrenia and control diagnostic groups (Figure 1c). We then proceeded with partial least squares discriminant analysis of the differentially expressed proteins to provide insight into the causes of discrimination between the diagnostic groups, and to pull out the proteins that were variables of importance (Figure 1d). Analysis of the variable importance plots from the partial least squares discriminant analysis revealed the most powerful discriminatory proteins between groups (Figure 1e), including elF2/4 pathway proteins (RPL14, RPL13A, RPL18A, PPP2R1A and FNRB), cytoskeletal proteins (FNRB, FLNA, C12orf8 and CDC10), mitochondrial proteins (TPI, LGALS1) and calcium-binding proteins (CALM and CAPL). Variable importance plots are ranked in order, where the protein that exhibits the strongest discriminatory power has the highest numerical value among the 20 descriptor variables (Figure 1e).

Notably, the uniform decreased expression of 17 ribosomal proteins $(P<0.05$; RPL4, RPL27A, RPL3, RPL27, RPL17, RPS6, RPL14, RPL9, RPL10A, RPL6, RPS4X, RPL18A, RPL32, RPS3, RPL13A, RPL13 and RPS14) was striking (Figure 2a). Analysis of the total MS signal intensity for all 67 cytoplasmic ribosome proteins identified found an $18 \%$ decrease in expression in the schizophrenia group $(P=0.0004$, likelihood-ratio test). In order to further confirm changes, we used an independent targeted MS approach ${ }^{12}$ to filter out target peptides of interest for relative quantification between groups. As the elF2a protein is known to regulate the initiation of protein synthesis, and was significantly implicated at the mRNA level in the same cells, ${ }^{10}$ we included it on our target list of proteins for extended validation. Single-ion monitoring ${ }^{12}$ was used to isolate and quantify target peptides of interest, and relative quantification between the pooled schizophrenia and control groups was performed. As it is not statistically valid to apply a Student's $t$-test to pooled data, the relative fold change between the diagnostic groups was used to confirm the direction of change of target proteins. This analysis demonstrated that the levels of elF2a, RPL13, RPL13A, RPL18A, RPL27A and RPL32 were reduced in schizophrenia patient-derived cells in

Figure 1. Protein expression profiling reveals discriminatory proteins between schizophrenia and control cell lines. (a) Line plots showing relative expression of the 859 protein profiled using label-free mass spectrometry (MS) across the 18 olfactory neurosphere-derived (ONS) cell lines, with overlaying box plots to illustrate the distribution and median of protein expression for each sample. (b) Volcano diagram plotting $t$-test difference versus significance $(-\log P$-value) for all proteins. Differentially expressed proteins $(P<0.05)$ are shown in turquoise and proteins significant following False Discovery Rate (FDR) correction are shown in red (see Supplementary Data set 2 for list). (c) Principal component analysis for differentially expressed proteins between the control (red circle) and schizophrenia (black circles) cell lines, $R^{2}=0.45$ for a three-component model. (d) Partial least squares discriminant analysis (PLS-DA) score plot for control (red circle) and schizophrenia (black circles) cell lines, with an $R^{2}=0.4$ and $Q^{2}=0.61$ for a three-component model. (e) PLS-DA table of the variables of importance (VIP) which were deemed as having the best discriminatory power of the 102 significant proteins tested (Supplementary Data set 2) for the control and schizophrenia diagnostic groups. These VIPs are ranked in order, whereby the protein that exhibits the strongest discriminatory power has the highest numerical value among the 20 descriptor variables. 
comparison with controls (Figure 2b). Significant reductions in elFa, RPL13A and RPL18A were also confirmed using western blot analysis on individual samples (see Supplementary Figure 1).
Global protein synthesis is reduced in schizophrenia ONS cells To address the question of whether reduced ribosomal protein levels lead to reduced rates of protein synthesis in patient-derived ONS cells, we quantified global protein synthesis rates using a

a

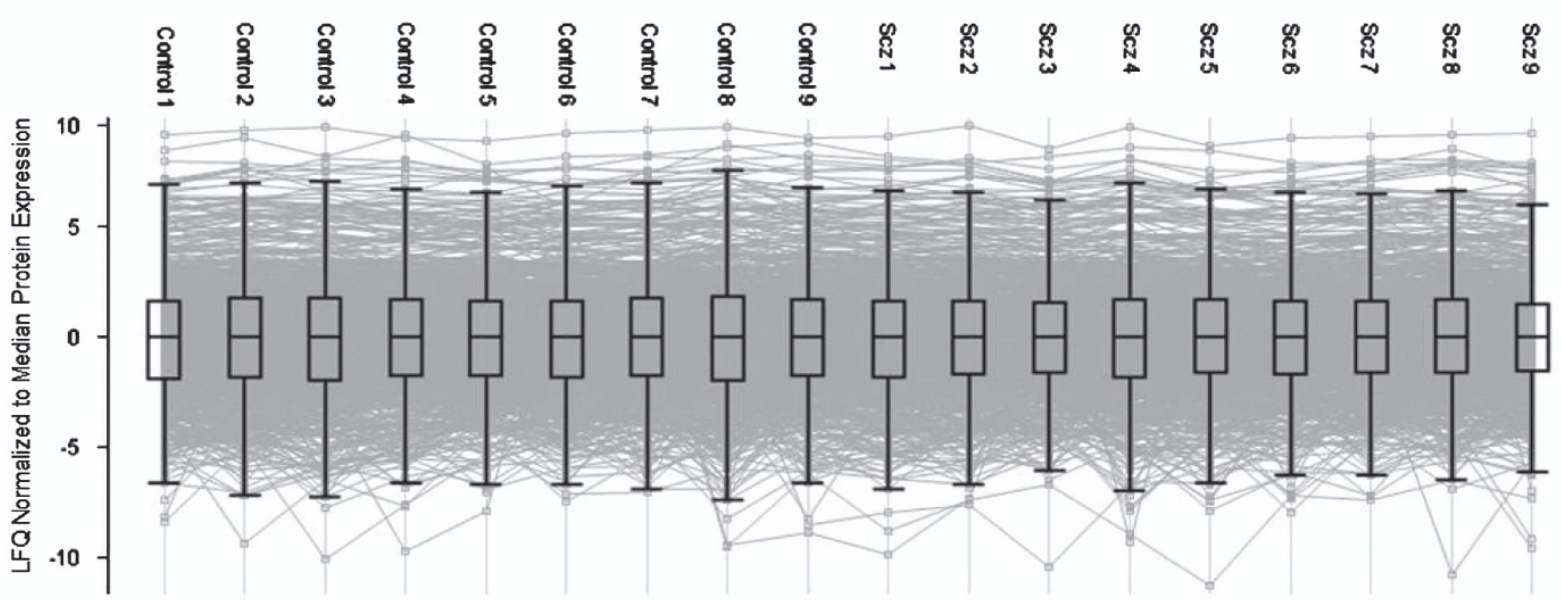

b

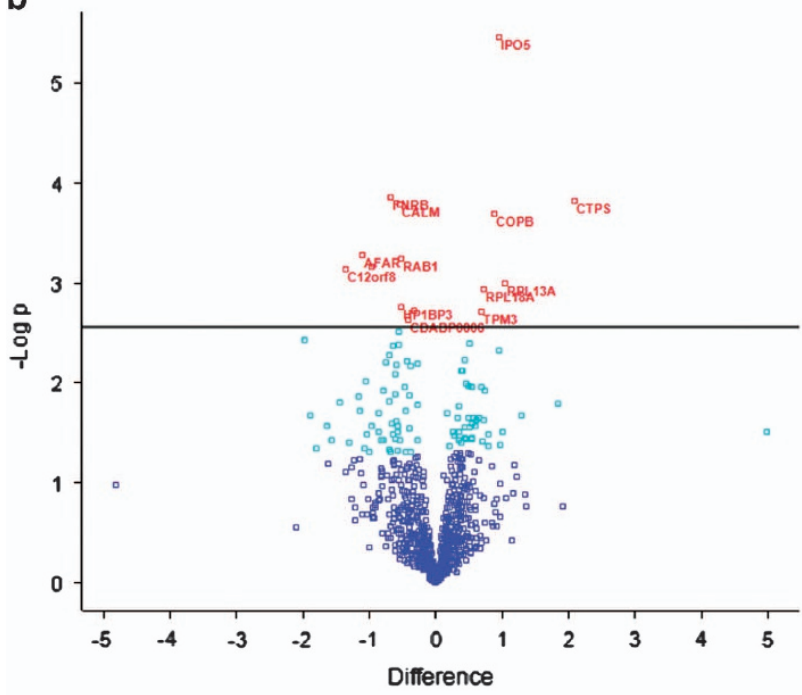

d

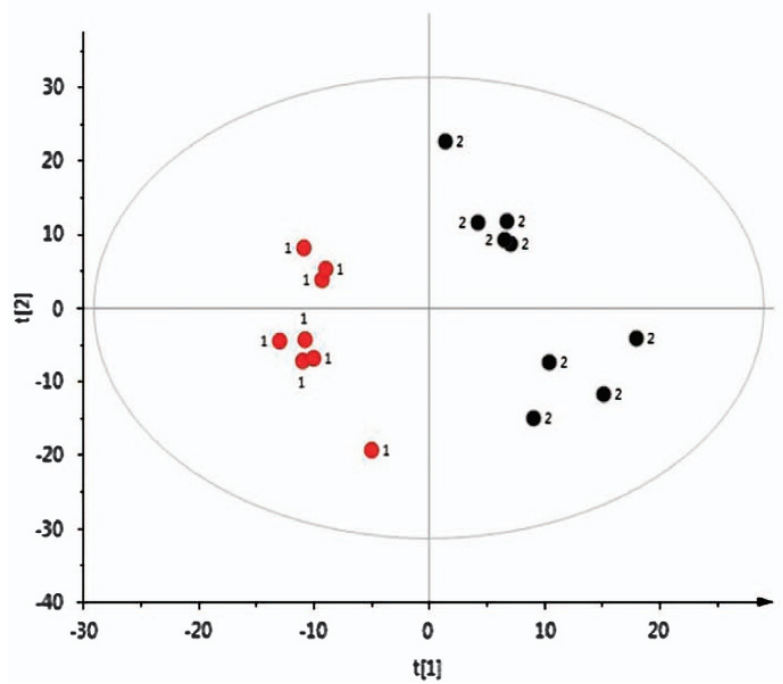

C

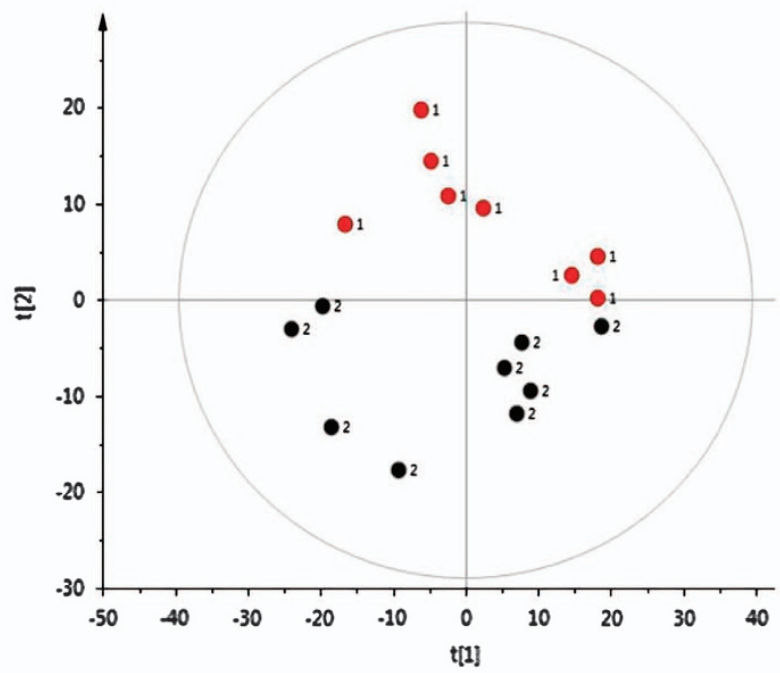

e

$\begin{array}{ll}\text { PLS-DA ID } & \text { VIP value } \\ \text { CALM } & 2.64628 \\ \text { IPO5 } & 2.64446 \\ \text { RPL14 } & 2.37504 \\ \text { CTPS } & 2.34843 \\ \text { FNRB } & 2.3481 \\ \text { COPB } & 2.29356 \\ \text { TPI } & 2.27421 \\ \text { RPL13A } & 2.21083 \\ \text { PPP2R1A } & 2.20088 \\ \text { AFAR } & 2.19691 \\ \text { RAB1 } & 2.19164 \\ \text { C12orf8 } & 2.18761 \\ \text { CCT1 } & 2.16145 \\ \text { LGALS1 } & 2.14884 \\ \text { CLEC13E } & 2.14717 \\ \text { DAAP-21F2.8-002 } & 2.12031 \\ \text { FLNA } & 2.11123 \\ \text { RPL18A } & 2.10359 \\ \text { CAPL } & 2.08996 \\ \text { CDC10 } & 2.08325\end{array}$




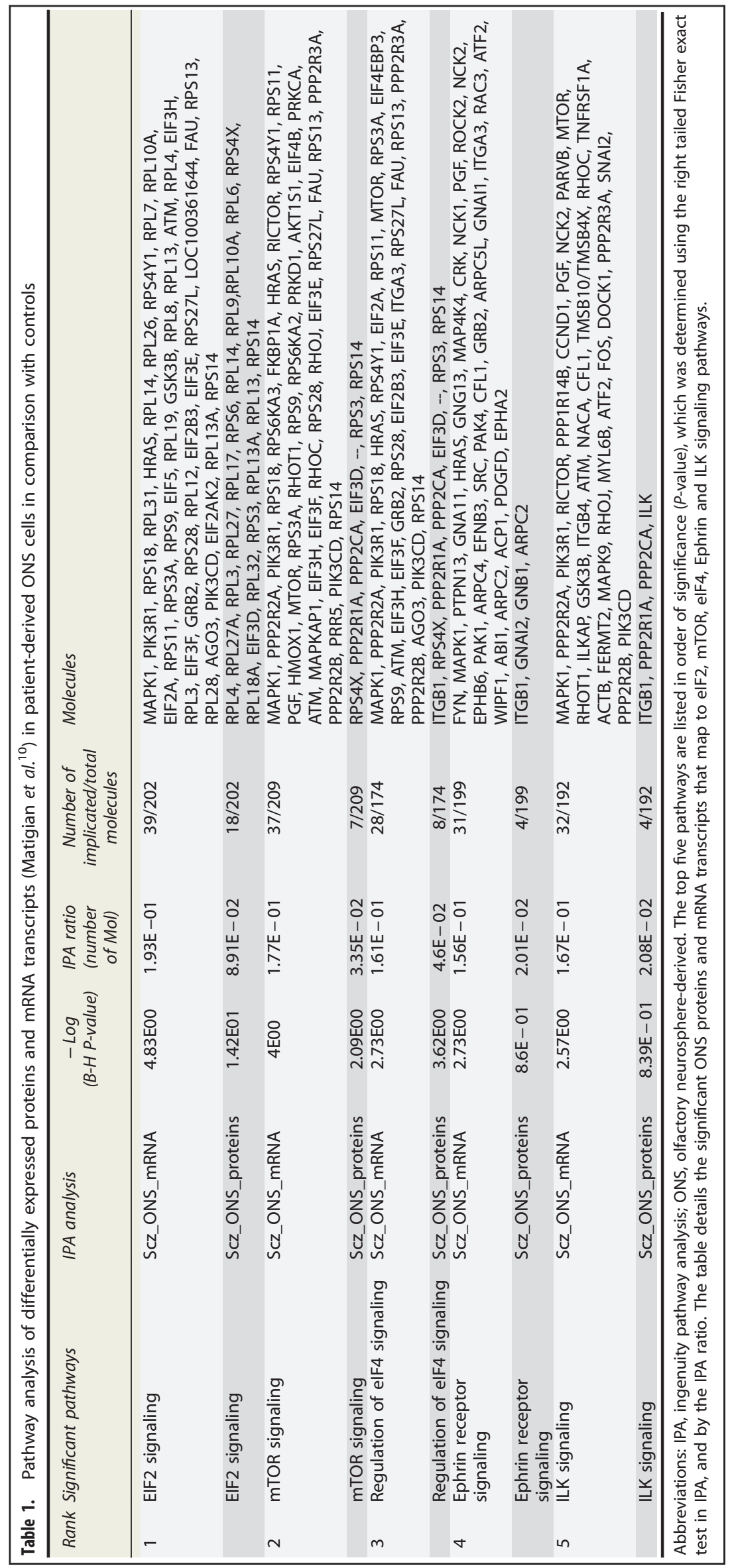


a
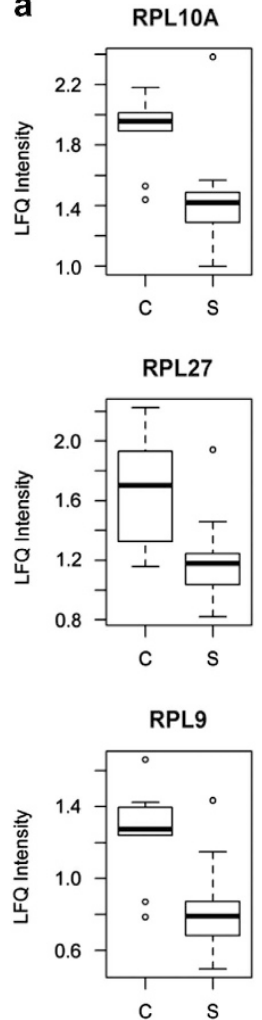

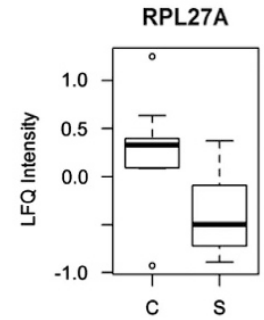

RPL13

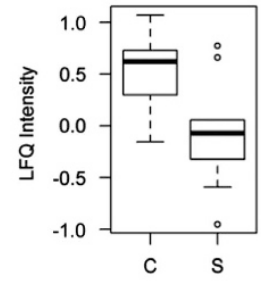

PL27A

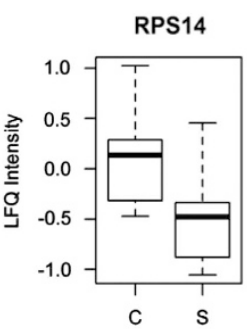

RPL13A

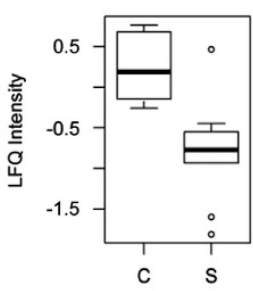

RPL3

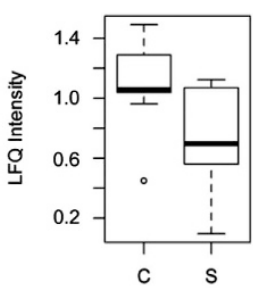

RPS3

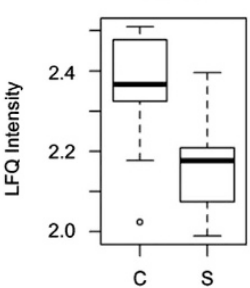

RPL14

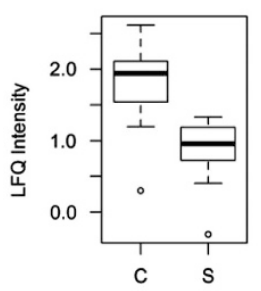

RPL32

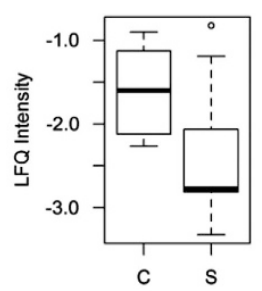

RPS4X

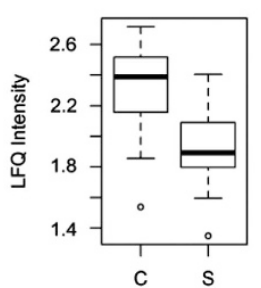

RPL17

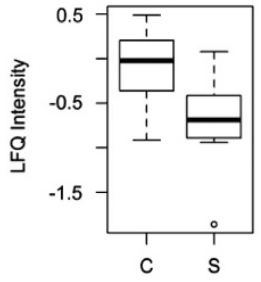

RPL4
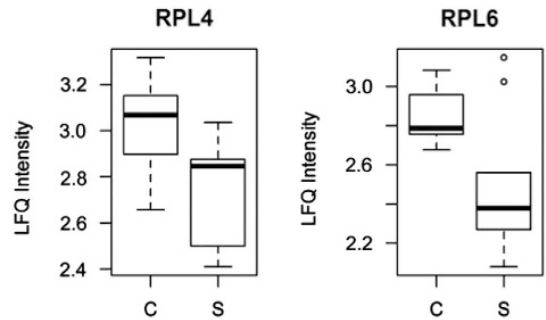

b

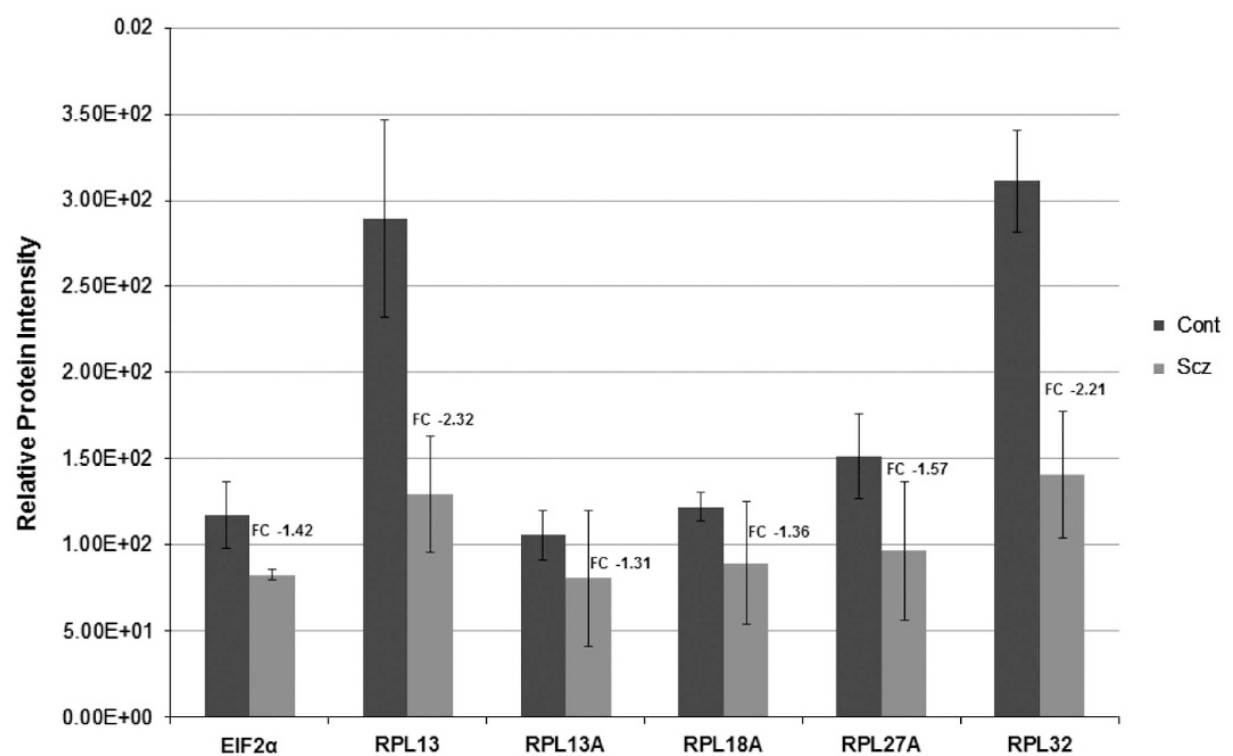

Figure 2. Reduced ribosomal protein expression in schizophrenia-derived olfactory neurosphere-derived (ONS) cells. (a) Boxplots representing the protein expression data from the discovery mass spectrometry (MS) analysis, which implicated 17 ribosomal proteins that were significantly decreased in expression in schizophrenia (S) patient-derived ONS cells compared with controls $(C ; P<0.05)$. The bottom and top of the boxes in each plot are the first (Q1) and third (Q3) quartiles, and the solid black band represents the median value or second quartile (Q2) for each RPL protein. The length of the rectangle from top to bottom is the interquartile range (IQR). The bottom whisker denotes either the minimum value or the first quartile minus 1.5 times the IQR $(\mathrm{Q} 1-1.5 * \mathrm{QQR})$, whichever is larger. The top whisker denotes the maximum value or the third quartile plus 1.5 times the IQR $\left(\mathrm{Q} 3+1.5^{*} \mathrm{IQR}\right)$, whichever is smaller. (b) We used targeted LC-MS analysis to isolate and quantify peptides of interest, in which we confirmed a relative decrease in expression of elF2 $\alpha$, RPL13, RPL13A, RPL18A, RPL27A and RPL32 proteins in pooled schizophrenia (Scz) ONS samples compared with pooled controls (Cont), which were run in triplicate. The relative intensity for each protein is represented on the $y$ axis and error bars denote \pm s.d. for each protein.

fluorescence incorporation assay for the methionine analog HPG. Experiments were performed on all nine schizophrenia patientderived and nine control-derived ONS cell lines, at three HPG concentrations, over four time points. Images were captured at three wavelengths to detect nuclei $(350 \mathrm{~nm}, 4,6$-diamidino-2phenylindole), cell cytoplasm $(647 \mathrm{~nm}$, CellMask) and newly synthetized protein (HPG, $488 \mathrm{~nm}$; Figure $3 a-c$, respectively). HPG fluorescence was quantified as the mean intensity per pixel 


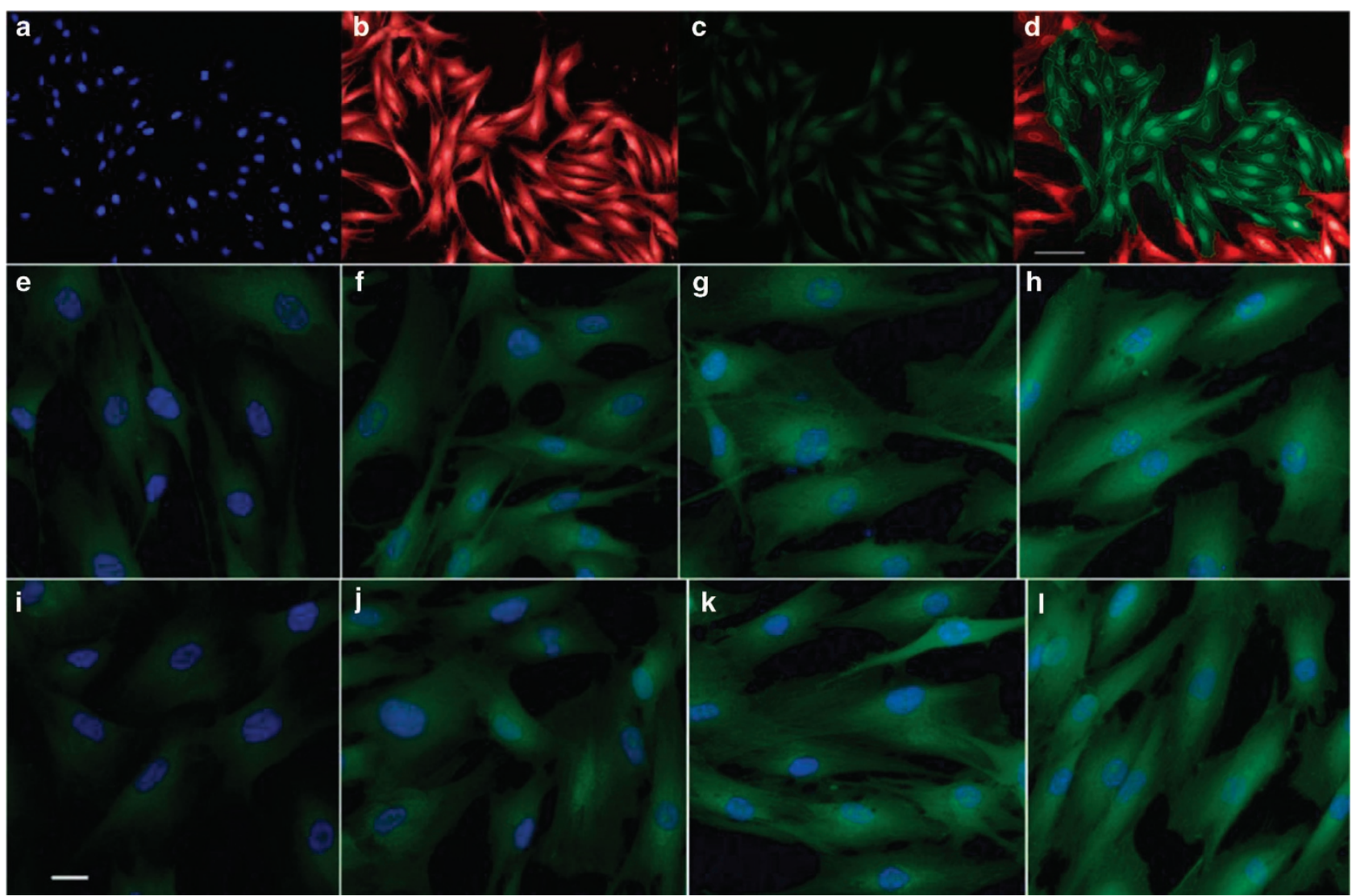

Figure 3. Quantification of global protein synthesis rates in schizophrenia patient-derived olfactory neurosphere (ONS)-derived cells. Automated quantification of global protein synthesis rates in ONS cell lines, and the same field of view for the nucleus ((a) 4',6-diamidino-2phenylindole (DAPI) stain), the cytoplasm ((b) CellMask stain), the L-homopropargylglycine (HPG) fluorescence ((c) azide fluorophore). (d) HPGpositive cells automatedly selected (green) and those discarded from the analysis because they overlapped the edge of the field (red). Control-derived ONS cells (e-h) and patient-derived ONS cells (i-I) were exposed for $1,2,4$ and $8 \mathrm{~h}$ to HPG at three concentrations ( 25 , 50 or $100 \mu \mathrm{m})$, with the $100 \mathrm{mM} \mathrm{HPG}$ concentration illustrated here. The scale bars are $100 \mu \mathrm{m}(\mathbf{a}-\mathbf{d})$ and $20 \mu \mathrm{m}$ (e-I). Global protein synthesis rates were measured in all 18 cell lines, for three HPG concentrations, for at least $8 \mathrm{~h}$.

per cell, and quantification was based on analysis of 255369 ONS cells across the nine patient-derived and nine control-derived cell lines. Although not obvious to the eye, schizophrenia-derived ONS cells were less fluorescent than control-derived ONS cells (Figure 3e-l), whereby ANOVA (Figure 4) found a significant effect for the disease status $\left(F_{1,396}=23.86, P<0.0001\right)$, confirming a schizophrenia-based effect on global protein synthesis, which was sustained for $8 \mathrm{~h}$. There were also significant effects of HPG concentration $\left(F_{2,396}=112.62, P<0.0001\right)$ and exposure time $\left(F_{3,396}=649.68, P<0.0001\right)$, indicating that, as expected, all cells increased their fluorescence when exposed to a higher concentration of HPG and for longer periods.

Linear regression was used to derive the rates of protein synthesis at each HPG concentration (Figure 4b), and the goodness of fit for these regression lines was estimated from the correlation coefficient, $R^{2}$, for each HPG concentration. The slopes of the regression lines were used in a linear regression to calculate Lineweaver-Burk plots (Figure $4 c ; R^{2}=1.00$ ), from which the rate constant $(\mathrm{Km})$ and maximum reaction velocity (Vmax) were calculated. The $\mathrm{Km}$ was higher for patient-derived ONS (54 versus $40 \mu \mathrm{M}$ ), and the $V \max$ was lower (95 versus $104 \mu \mathrm{M} \mathrm{h}-1$ ), in comparison with control-derived ONS cells, confirming that the control cells had a faster rate of protein synthesis (smaller $\mathrm{Km}$ ) and larger capacity for protein synthesis (larger Vmax). ANOVA of the Lineweaver-Burk regressions was significant $\left(F_{1,2}=79.98\right.$, $P=0.012$ ), indicating that the slopes were significantly different.
We then asked whether the reduction in protein synthesis was cell-type-specific by quantifying protein synthesis rates in fibroblasts from the same individuals, and found that control and schizophrenia patient-derived fibroblasts were of similar fluorescence (Figure 4d-f). Furthermore, ANOVA demonstrated no significant effect of the disease status $\left(F_{1,312}=0.561, P=0.454\right)$. To address the question of whether reduced protein synthesis is correlated with reduced cell size, cell area was quantified using the cytoplasmic CellMask stain. This analysis found that schizophrenia patient-derived ONS cells were smaller than control-derived cells $\left(2671+42\right.$ versus $3687+61 \mu \mathrm{m}^{2} ; F_{1,396}=$ 184.75; $P<0.0001)$. In contrast, schizophrenia patient-derived fibroblasts were larger than control-derived fibroblast cells (2650 +95 versus $\left.2151+29 \mu \mathrm{m}^{2} ; \mathrm{F}_{1,336}=27.33 ; P<0.0001\right)$. Overall, these findings demonstrate that the reduced expression of ribosomal proteins in schizophrenia patient-derived ONS cells was associated with a significant reduction in the rate of protein synthesis compared with ONS cells from healthy controls.

Upstream translational control pathways elF2/4 and mTOR signaling are dysregulated in patient-derived ONS and iPSC models

As mRNA and protein expression levels are biochemically linked, we integrated our proteomic data with transcriptomic mRNA data from the same ONS cell lines using the Ingenuity Pathway Analysis tool. ${ }^{10}$ Our objective was to identify disrupted protein pathways, 

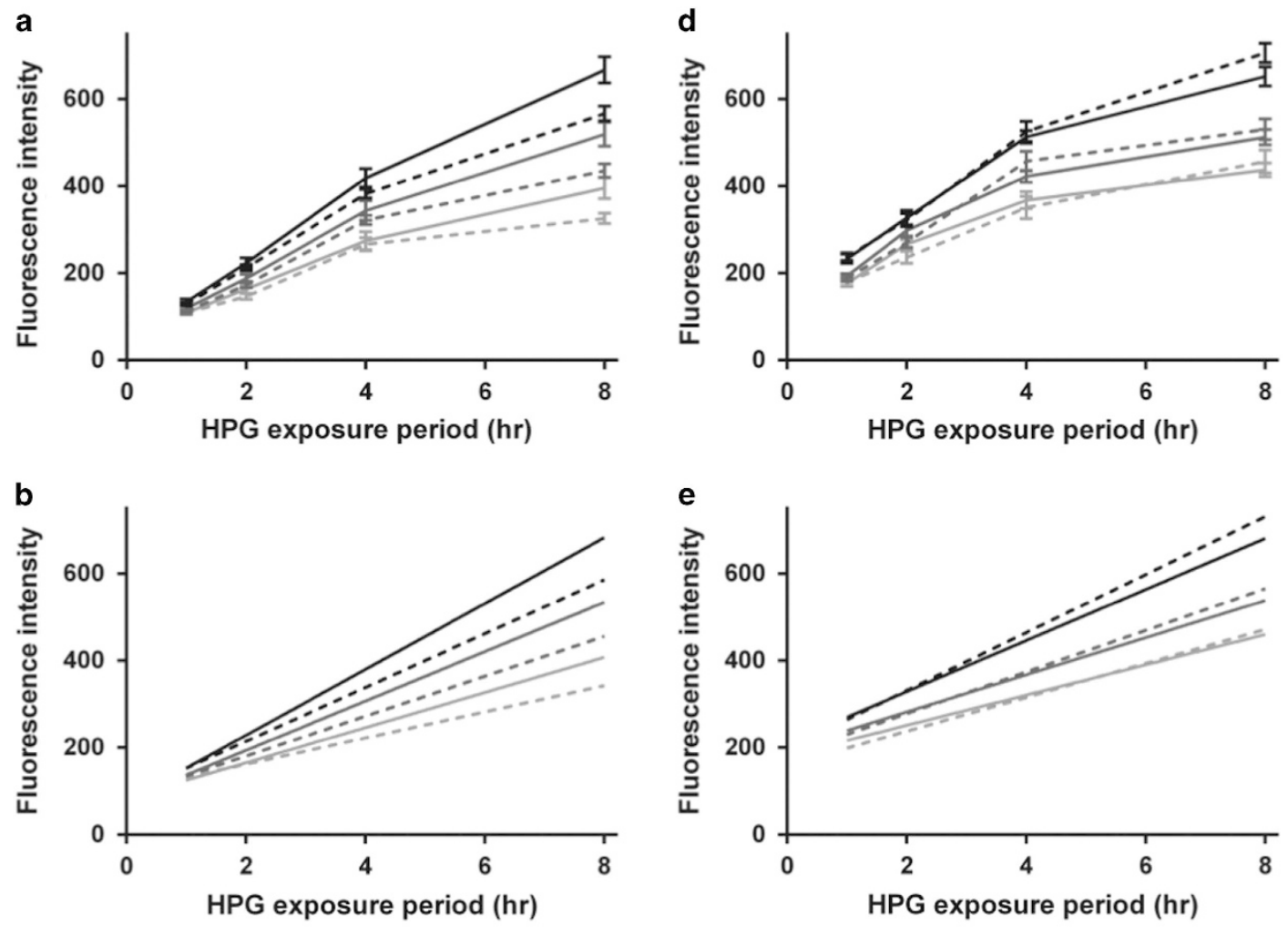

c

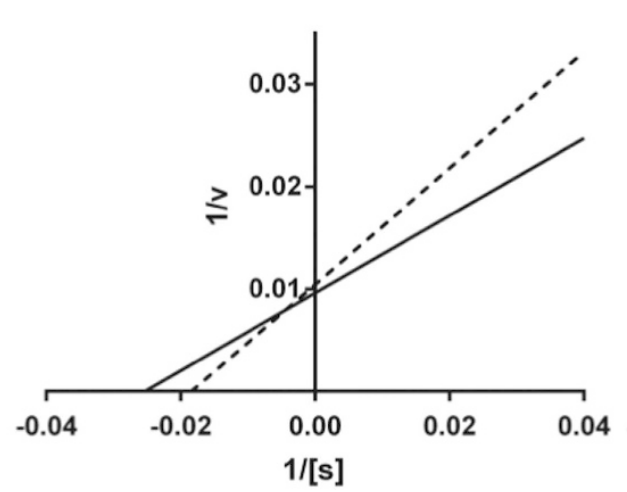

f

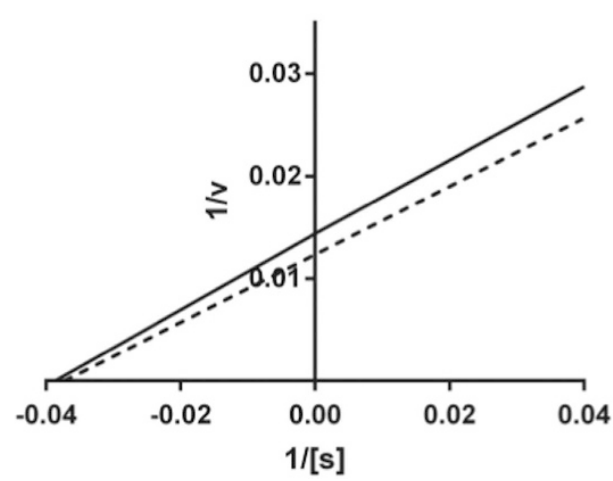

Figure 4. Reduced rates of global protein synthesis in schizophrenia patient-derived olfactory neurosphere-derived (ONS) cells in comparison with controls. Regression model illustrating global protein synthesis was reduced in nine patient-derived cell lines in comparison with nine control cell lines, but not in fibroblasts from the same patients. (a) L-homopropargylglycine (HPG) fluorescence at 1-, 2-, 4- and 8-h exposure periods in control-derived ONS cells (lines) and patient-derived ONS cells (dashed lines) at different HPG concentrations (100 $\mu \mathrm{m}$, black; $50 \mu \mathrm{M}$, mid-gray; $25 \mu \mathrm{m}$, light gray). (b) Linear regression of the HPG fluorescence shown in a. (c) Lineweaver-Burk plots for the global protein synthesis reactions in control-derived ONS cells (line) and patient-derived ONS cells (dashed line) calculated from the slopes of the regression lines shown in $\mathbf{b}$. The slopes of these lines are significantly different from each other $\left(\mathrm{F}_{1,2}=79.98, P=0.012, R^{2}=0.94\right)$. (d-f) Results for global protein synthesis rates in control-derived fibroblasts (line) and patient-derived fibroblasts (dashed line) from the same patients. The slopes of these lines were not significantly different from each other $\left(F_{1,2}=0.0707, P=0.815, R^{2}=0.94\right)$.

rather than single proteins; therefore, we uploaded the full list 102 significant proteins $(P<0.05)$ for pathway analysis (Supplementary Data set 2). The most significant molecular pathways implicated (Benjamini-Hochberg FDR-adjusted Fisher's exact test at $P<0.01$ ) in both protein and mRNA data sets were elF2 signaling, mTOR signaling and elF4 signaling (Table 1; Supplementary Data set 3), all of which are upstream pathways concerned with the regulation of translational control and protein synthesis. ${ }^{16}$ No effects of smoking status and antipsychotic dose on protein expression were observed (data not shown). We next examined proteomic expression data from an independent study by Brennand et al., ${ }^{9}$ asking whether the elF2/4 and mTOR pathways were also dysregulated in schizophrenia patient-derived neuronal precursor cells generated from iPSCs. Pathway analysis of this proteomic data set confirmed that elF2/4 and mTOR signaling were again the most significant pathways implicated (Benjamini-Hochberg FDR-adjusted Fisher's exact test at $P<0.01$; Supplementary Data set 4).

Protein synthesis implicated in schizophrenia by genome-wide association studies

Finally, we reasoned that if the genes involved in elF2 signaling are involved in the pathology of schizophrenia, then this may be 
reflected in population-based clinical studies. We tested this by performing gene-based association analysis, ${ }^{17}$ with molecules implicated in elF2 signaling (corresponding to proteins and mRNA transcripts implicated in elF2 signaling; Table 1) using genomewide association data based on $>13000$ schizophrenia cases and $>18000$ controls. $^{14}$ Two proteins showed a significant association with schizophrenia, EIF2AK2 $\left(P=3 \times 10^{-6}\right)$ and RPS13 $(P=0.00029)$, following Bonferroni correction ( $P$-value $0.05 / 48$ genes tested $=$ significance required $P<0.0010$; Supplementary Data set 5$)$. EIF2AK2 had the most significant gene-based score in the chromosome 2 p22 region, whereas the neighboring gene to RPS13, PIK3C2A, is more significantly associated with schizophrenia $(P=0.000126)$ than RPS13, thus not allowing us to verify the specific source of the RPS13 association signal from this analysis (data not shown).

We then questioned whether all three biological pathways concerned with translational control-elF2, mTOR and elF4 signaling-were implicated in schizophrenia genomics data. We tested this by performing 'Gene-set Enrichment Analysis' ${ }^{\prime 5}$ with molecules implicated in translational control (corresponding to the elF2, elF44 and mTOR proteins and mRNA transcripts in Table 1) in genome-wide association data, ${ }^{14}$ and the effect was significantly associated with schizophrenia following Bonferroni correction $(P=0.019$; Supplementary Data set 5$)$, and showed a trend association with bipolar disorder $(P=0.084)$, but not Crohn's disease or type 2 diabetes (Supplementary Data set 5).

\section{DISCUSSION}

We used a proteomics expression profiling approach to identify co-regulated protein pathways disrupted in schizophrenia patientderived ONS cells in comparison with controls. Of the 102 proteins that were differentially expressed, 17 were ribosomal proteins that were significantly decreased, and several ribosomal/elF2 signaling proteins (RPL14, RPL13A, RPL18A, PPP2R1A and FNRB) were robust discriminators between the control and schizophrenia groups (Figure 1). Led by these findings, we quantified global protein synthesis rates and demonstrated that patient-derived cell lines had significantly reduced rates of protein synthesis compared with control-derived cell lines. In addition, data integration analyses showed that the protein expression data converged with mRNA expression, and genome-wide association studies highlighted dysregulated elF2a, mTOR and elF4 signaling pathways, as correlating with the disease status.

Our findings of reduced cell area, ribosomal protein expression and rates of protein synthesis are consistent with previous postmortem studies in schizophrenia, showing reduced neuronal somal size ${ }^{18,19}$ and imaging studies that report reductions in whole-brain volume ${ }^{20}$ in schizophrenia. In keeping with the current study, we also recently reported altered ribosomal protein expression (RPLP1, RPLP2, RPL30 and RPL19) and Cytoplasmic FMR1 Interacting Protein 2 (CYFIP2) in post-mortem brain in schizophrenia. ${ }^{21}$ CYFIP regulates protein synthesis at the synapse by coordinating local mRNA translation and cytoskeleton remodeling to ensure proper dendritic spine formation. ${ }^{22-24}$ Previous stem cell studies of schizophrenia reported altered cellular migration and motility, 7,9 and our findings of altered expression of proteins involved in cytoskeletal remodeling (ARPC2, CYFIP2, GNAI2, GNB1, ITGB1, PDIA3, PFN2 and TLN1) and cellular adhesion and migration (ILK, ITGB1, PPP2CA, PPP2R1A, ITGB1, GMAI2, GNB1 and ARPC2) are consistent with these studies, and with previous post-mortem brain studies of schizophrenia. ${ }^{25,26}$

The upstream signaling pathways concerned with translational control of protein synthesis are elF2 and mTOR signaling, ${ }^{16}$ and we observed these pathways to be dysregulated in schizophrenia patient-derived ONS and iPSC models, ${ }^{9}$ and at the level of the genome in schizophrenia population-based data. In particular, elF2 signaling is central to synaptic plasticity, learning and memory, ${ }^{27}$ and, given that genes involved in synaptic function are strongly implicated in schizophrenia risk, ${ }^{28,29}$ it is reasonable to propose that dysregulation of protein synthesis via elF2 signaling may contribute to schizophrenia. We tested the association of the 48 differentially expressed elF2 pathway proteins and mRNA transcripts in a genome-wide association studies of $>13000$ schizophrenia patients ${ }^{14}$ and found that 15 elF2 pathway proteins reached significance, and two (EIF2AK2 and RPS13) were highly significantly associated with the schizophrenia phenotype. In particular, EIF2AK phosphorylates elF2a in response to viral infection, which in turn blocks the translation of viral mRNAs and protein synthesis. ${ }^{30,31}$ This is particularly relevant, given the current literature implicating prenatal infection and inflammation as risk factors for schizophrenia. ${ }^{32,33}$

The mTOR and elF4 signaling pathways are also critical regulators of protein synthesis and ribosomal biogenesis, and have been previously implicated in neuropsychiatric disorders. ${ }^{34}$ Through phosphorylation of its downstream elF4-binding proteins, mTOR complex 1 promotes protein synthesis, ${ }^{34}$ and direct pharmacological inhibition of mTORC1 blocks L-LTP and LTM. ${ }^{35,36}$ Moreover, the antipsychotic medication haloperidol was recently shown to activate mTORC 1 and enhance protein synthesis via elF4 signaling in cultured neurons. ${ }^{37}$ In this context, it is important to note that regulation of protein synthesis may be cell-typedependent. We observed deficits in protein synthesis rates in ONS cells but not in fibroblasts from the same patients, and these findings are supported by previous literature (for review see ref. 38). Given the potential disruption of all three translational control mechanisms elF2, mTor and elF4 signaling, which ultimately converge on altered protein synthesis, we performed gene-set enrichment analysis to test the association of these pathways in genome-wide association studies from individuals with schizophrenia and found a significant association with the schizophrenia phenotype.

In summary, our study has integrated data from proteomic, transcriptomic and in vitro functional assays in schizophreniapatient-derived ONS cells, and genomic analyses, to provide important evidence that disturbed protein synthesis is associated with schizophrenia and could contribute to the development of the disorder. Future work is required to elucidate the specificity of these changes in the context of other neuropsychiatric disorders, and to determine the consequences of dysregulated protein synthesis at different developmental stages and in different cell types (for example, neurons versus glial cells during development). Overall, these data point to the dysregulation of protein synthesis in schizophrenia and suggest new molecular targets for intervention.

\section{CONFLICT OF INTEREST}

The authors declare no conflict of interest.

\section{ACKNOWLEDGMENTS}

This work was supported by the Health Research Board Clinical Scientist Award and 10/RFP/NES2744 (DRC). The work was also supported by a grant from the Australian Government Department of Health and Ageing (AM-S). We thank Dr Aoife O'Gorman, RCSI and UCD Conway Institute for statistical input, and gratefully acknowledge Dr Kristen Brennand, Icahn Medical Institute Mount Sinai, for helpful discussions while preparing this manuscript.

\section{REFERENCES}

1 Murray CJ, Lopez AD. Global mortality, disability, and the contribution of risk factors: global burden of disease study. Lancet 1997; 349: 1436-1442.

2 Mackay-Sim A. Concise review: patient-derived olfactory stem cells: new models for brain diseases. Stem Cells 2012; 30: 2361-2365.

3 Horiuchi Y, Kano S, Ishizuka K, Cascella NG, Ishii S, Talbot CC Jr et al. Olfactory cells via nasal biopsy reflect the developing brain in gene expression profiles: utility 
and limitation of the surrogate tissues in research for brain disorders. Neurosci Res 2013; 77: 247-250.

4 Toritsuka M, Kimoto S, Muraki K, Landek-Salgado MA, Yoshida A, Yamamoto N et al. Deficits in microRNA-mediated $\mathrm{Cxcr} 4 / \mathrm{Cxcl} 12$ signaling in neurodevelopmental deficits in a 22q11 deletion syndrome mouse model. Proc Natl Acad Sci USA 2013; 110: 17552-17557.

5 Kano S, Colantuoni C, Han F, Zhou Z, Yuan Q, Wilson A et al. Genome-wide profiling of multiple histone methylations in olfactory cells: further implications for cellular susceptibility to oxidative stress in schizophrenia. Mol Psychiatry 2013; 18: $740-742$.

6 Mor E, Kano S, Colantuoni C, Sawa A, Navon R, Shomron N. MicroRNA-382 expression is elevated in the olfactory neuroepithelium of schizophrenia patients. Neurobiol Dis 2013; 55: 1-10.

7 Fan Y, Abrahamsen G, Mills R, Calderon CC, Tee JY, Leyton L et al. Focal adhesion dynamics are altered in schizophrenia. Biol Psychiatry 2013; 74: 418-426.

8 Fan Y, Abrahamsen G, McGrath JJ, Mackay-Sim A. Altered cell cycle dynamics in schizophrenia. Biol Psychiatry 2012; 71: 129-135.

9 Brennand K, Savas JN, Kim Y, Tran N, Simone A, Hashimoto-Torii K et al. Phenotypic differences in hiPSC NPCs derived from patients with schizophrenia. $\mathrm{Mol}$ Psychiatry 2014; 20: 361-368.

10 Matigian N, Abrahamsen G, Sutharsan R, Cook AL, Vitale AM, Nouwens A et al. Disease-specific, neurosphere-derived cells as models for brain disorders. Dis Model Mech 2010; 3: 785-798.

11 English JA, Harauma A, Föcking M, Wynne K, Scaife C, Cagney G et al. Omega-3 fatty acid deficiency disrupts endocytosis, neuritogenesis, and mitochondrial protein pathways in the mouse hippocampus. Front Genet 2013; 4: 208.

12 Schilling B, Rardin MJ, MacLean BX, Zawadzka AM, Frewen BE, Cusack MP et al. Platform-independent and label-free quantitation of proteomic data using MS1 extracted ion chromatograms in skyline: application to protein acetylation and phosphorylation. Mol Cell Proteomics 2012; 11: 202-214.

13 Escher C, Reiter L, MacLean B, Ossola R, Herzog F, Chilton J et al. Using iRT, a normalized retention time for more targeted measurement of peptides. Proteomics 2012; 12: 1111-1121.

14 Ripke S, O'Dushlaine C, Chambert K, Moran JL, Kahler AK, Akterin S et al. Genomewide association analysis identifies 13 new risk loci for schizophrenia. Nat Genet 2013; 45: 1150-1159.

15 Wang K, Li M, Hakonarson H. Analysing biological pathways in genome-wide association studies. Nat Rev Genet 2010; 11: 843-854.

16 Buffington SA, Huang W, Costa-Mattioli M. Translational control in synaptic plasticity and cognitive dysfunction. Annu Rev Neurosci 2014; 37: 17-38.

17 Liu JZ, McRae AF, Nyholt DR, Medland SE, Wray NR, Brown KM et al. A versatile gene-based test for genome-wide association studies. Am J Hum Genet 2010; 87: 139-145.

18 Rajkowska G, Selemon LD, Goldman-Rakic PS. Neuronal and glial somal size in the prefrontal cortex: a postmortem morphometric study of schizophrenia and Huntington disease. Arch Gen Psychiatry 1998; 55: 215-224.

19 Chana G, Landau S, Beasley C, Everall IP, Cotter D. Two-dimensional assessment of cytoarchitecture in the anterior cingulate cortex in major depressive disorder, bipolar disorder, and schizophrenia: evidence for decreased neuronal somal size and increased neuronal density. Biol Psychiatry 2003; 53: 1086-1098.

20 Harrison PJ, Weinberger DR. Schizophrenia genes, gene expression, and neuropathology: on the matter of their convergence. Mol Psychiatry 2005; 10: 40-68, image 45 .
21 Focking M, Lopez LM, English JA, Dicker P, Wolff A, Brindley E et al. Proteomic and genomic evidence implicates the postsynaptic density in schizophrenia. Mol Psychiatry 2014; 20: 424-432.

22 De Rubeis S, Pasciuto E, Li KW, Fernandez E, Di Marino D, Buzzi A et al. CYFIP1 coordinates mRNA translation and cytoskeleton remodeling to ensure proper dendritic spine formation. Neuron 2013; 79: 1169-1182.

23 Yoon KJ, Nguyen HN, Ursini G, Zhang F, Kim NS, Wen Z et al. Modeling a genetic risk for schizophrenia in iPSCs and mice reveals neural stem cell deficits associated with adherens junctions and polarity. Cell Stem Cell 2014; 15: 79-91.

24 Abekhoukh S, Bardoni B. CYFIP family proteins between autism and intellectual disability: links with Fragile X syndrome. Front Cell Neurosci 2014; 8: 81.

25 English JA, Dicker P, Focking M, Dunn MJ, Cotter DR. 2-D DIGE analysis implicates cytoskeletal abnormalities in psychiatric disease. Proteomics 2009; 9: 3368-3382.

26 English JA, Pennington K, Dunn MJ, Cotter DR. The neuroproteomics of schizophrenia. Biol Psychiatry 2011; 69: 163-172.

27 Costa-Mattioli M, Sossin WS, Klann E, Sonenberg N. Translational control of longlasting synaptic plasticity and memory. Neuron 2009; 61: 10-26.

28 Fromer M, Pocklington AJ, Kavanagh DH, Williams HJ, Dwyer S, Gormley P et al. De novo mutations in schizophrenia implicate synaptic networks. Nature 2014; 506: 179-184.

29 Pocklington AJ, O'Donovan M, Owen MJ. The synapse in schizophrenia. Eur J Neurosci 2014; 39: 1059-1067.

30 Donnelly N, Gorman AM, Gupta S, Samali A. The elF2alpha kinases: their structures and functions. Cell Mol Life Sci 2013; 70: 3493-3511.

31 Rothenburg S, Seo EJ, Gibbs JS, Dever TE, Dittmar K. Rapid evolution of protein kinase PKR alters sensitivity to viral inhibitors. Nat Struct Mol Biol 2009; 16: 63-70.

32 Carter CS, Bullmore ET, Harrison P. Is there a flame in the brain in psychosis? Biol Psychiatry 2014; 75: 258-259.

33 Meyer U. Prenatal poly(i:C) exposure and other developmental immune activation models in rodent systems. Biol Psychiatry 2014; 75: 307-315.

34 Costa-Mattioli M, Monteggia LM. mTOR complexes in neurodevelopmental and neuropsychiatric disorders. Nat Neurosci 2013; 16: 1537-1543.

35 Tang SJ, Reis G, Kang H, Gingras AC, Sonenberg N, Schuman EM. A rapamycinsensitive signaling pathway contributes to long-term synaptic plasticity in the hippocampus. Proc Natl Acad Sci USA 2002; 99: 467-472.

36 Stoica L, Zhu PJ, Huang W, Zhou H, Kozma SC, Costa-Mattioli M. Selective pharmacogenetic inhibition of mammalian target of Rapamycin complex I (mTORC1) blocks long-term synaptic plasticity and memory storage. Proc Natl Acad Sci USA 2011; 108: 3791-3796.

37 Bowling H, Zhang G, Bhattacharya A, Perez-Cuesta LM, Deinhardt K, Hoeffer CA et al. Antipsychotics activate mTORC1-dependent translation to enhance neuronal morphological complexity. Sci Signal 2014; 7: ra4.

38 Buszczak M, Signer RA, Morrison SJ. Cellular differences in protein synthesis regulate tissue homeostasis. Cell 2014; 159: 242-251.

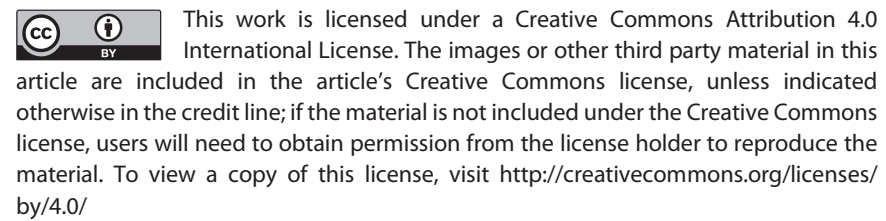
by/4.0/

Supplementary Information accompanies the paper on the Translational Psychiatry website (http://www.nature.com/tp) 